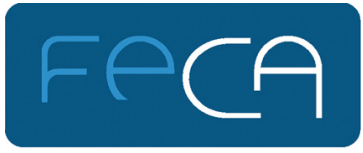

Journal of Healthcare Quality Research

\title{
ORIGINAL
}

\section{Percepción de la calidad de la investigación enfermera en un hospital público}

\author{
Y.G. Santana Padilla ${ }^{a}$, J.D. Martin Santana ${ }^{b}$, L. Santana-Cabrera ${ }^{a, *}$ y M.D. Bernat Adell ${ }^{c}$ \\ a Complejo Hospitalario Universitario Insular Materno Infantil, Las Palmas de Gran Canaria, España \\ b Universidad de Las Palmas de Gran Canaria, Las Palmas de Gran Canaria, España \\ c Universitat Jaume I, Castellón de la Plana, España
}

Recibido el 19 de julio de 2017; aceptado el 6 de marzo de 2018

\author{
PALABRAS CLAVE \\ Investigación \\ en enfermería; \\ Investigación en \\ servicios de salud; \\ Enfermería; \\ Evaluación; \\ Investigación \\ biomédica
}

\begin{abstract}
Resumen
Antecedentes y objetivos: Las enfermeras, como profesionales sanitarios, juegan un papel importante en la investigación, ya que sin ellas no se podría avanzar en el cuidado y en los tratamientos. Por ello nos planteamos como objetivos: primero, analizar la percepción de la enfermería sobre el actual sistema de investigación en el conjunto de su hospital y, segundo, estudiar cómo varía dicha percepción en función de su perfil investigador y de su nivel de satisfacción y compromiso con la organización en la que trabaja.

Material y método: Estudio descriptivo transversal, realizado en un centro hospitalario de tercer nivel. Se trabajó con una muestra de conveniencia formada por enfermeras con una experiencia superior a 6 meses. Se utilizó el cuestionario del Grupo de Expertos del III Foro de Ciencia de la Fundación Lilly, adaptándolo a las características de la población. La encuesta tenía por objeto recoger la percepción y valoración, real e ideal, sobre la investigación que tiene este colectivo. Se realizaron análisis univariados y bivariados mediante el estadístico $t$ de Student. Resultados: Para una muestra formada por 295 enfermeras, la percepción y valoración de la situación actual de la investigación en enfermería, el impacto de esta, su reconocimiento y su integración con la labor asistencial estuvieron muy por debajo de las puntuaciones que consideran ideales, obteniéndose diferencias estadísticamente significativas $(p<0,001)$. Resultaron significativos los valores que reconocen que se precisa mayor investigación por parte de la enfermería, el impacto y reconocimiento por la gerencia y la sinergia con la industria farmacéutica $(p<0,001)$. El nivel de satisfacción no influyó en la valoración, a diferencia del compromiso. No se observaron diferencias entre el personal de la $\mathrm{UCI}$ respecto del resto de servicios en cuanto a la percepción y valoración de la investigación de la enfermería.

Conclusiones: Las enfermeras asumen la investigación como parte de sus funciones y afirman que el estado de la investigación es muy mejorable. Según estas, es necesaria una infraestructura de apoyo que potencie la investigación en cuidados y el reconocimiento real por parte de las instituciones.
\end{abstract}

(c) 2018 FECA. Publicado por Elsevier España, S.L.U. Todos los derechos reservados.

\footnotetext{
* Autor para correspondencia.

Correo electrónico: Isancabx@gobiernodecanarias.org (L. Santana-Cabrera).
} 


\section{KEYWORDS}

Nursing research;

Health services

research;

Nursing;

Evaluation;

Biomedical research

\section{Perception of the quality of nursing research in a public hospital}

\begin{abstract}
Background and objectives: Nurses, as health professionals, play an important role in research, as progress in care and treatment could not be made without it. The aim of this study is to analyse the perception by nurses of the current research system in the whole of their hospital and, second, study how this perception varies according to their research profile, as well as their level of satisfaction and commitment to the organisation in which work.

Material and method: A cross-sectional descriptive study was conducted in a third level hospital with a convenience sample of nurses with more than 6 months experience. The Group of Experts of the III Forum of Science of the Lilly Foundation questionnaire was used, adapting it to the characteristics of the population. The purpose of the questionnaire was to collect the perception and assessment, real and ideal, that nurses have on research. Univariate and bivariate analyses were performed using the Student $t$-test.

Results: In the sample of 295 nurses, the perception and assessment of the current situation of nursing research, its impact, its recognition, and its integration with nursing work were well below the ideal scores, obtaining statistically differences $(P<.001)$. There was a significance in the values that recognise that more research is needed by nursing staff, the impact and recognition by management, and synergy with the pharmaceutical industry $(P<.001)$. The level of satisfaction did not affect the assessment, the commitment of the nurses if it influenced their assessment of the research. No differences were found between the ICU staff and the other departments as regards the perception and assessment of nursing research.

Conclusions: Nurses take the research as part of their functions and mention that the state of the research is very much improved. A support infrastructure is needed to strengthen research in care, as well as real recognition by institutions.
\end{abstract}

(c) 2018 FECA. Published by Elsevier España, S.L.U. All rights reserved.

\section{Introducción}

Todos los autores están de acuerdo en que es necesario un desarrollo en la investigación sanitaria en España. Martín-Moreno et al. ${ }^{1}$ afirman que se deben establecer estrategias coordinadas entre todos los sectores implicados para generar un desarrollo científico homogéneo y estable en el tiempo, para lo cual se deben cumplir las normativas articuladas por el Estado para ello ${ }^{2-4}$.

Según Puerta et al. ${ }^{5}$, el estado actual de la investigación biomédica, desde la perspectiva de los investigadores, si bien ha mejorado en las últimas décadas, en determinados centros solo es posible desarrollar ciertos tipos de proyectos de investigación y en algunos ni siquiera se dan las mínimas condiciones. Esta situación extiende la creencia, como señala Soriguer ${ }^{6}$, de que la investigación clínica es la gran olvidada del sistema sanitario, lo que puede entrar en contradicción con la percepción de que sea bueno, ya que la calidad está vinculada con la investigación. Y es que, como bien apuntan Pons et al. ${ }^{7}$, los índices de calidad de los hospitales pueden relacionarse con la investigación e índices de publicación de los distintos centros y servir para comparar hospitales en el mismo país.

Las dificultades para adoptar una cultura científica en el ambiente asistencial pueden ser múltiples; autores como Miró et al. ${ }^{8}$, Vento Torres et al. ${ }^{9}$ y Font et al. ${ }^{10}$ consideran que la falta de formación metodológica o el apoyo de la institución son dos elementos fundamentales para lograr culminar con éxito cualquier iniciativa de naturaleza investigadora. Es, por tanto, una necesidad prioritaria la presencia de estructuras organizativas destinadas a tal fin. Se puede citar como ejemplo de éxito la creación del programa de posgrado de la Harvard School of Public Health destinado a fomentar la investigación entre médicos noveles ${ }^{11}$.

A pesar de estas dificultades, puede afirmarse que la investigación biomédica ha mejorado sustancialmente en España, como así lo indican los trabajos de bibliometría llevados a cabo por Camí et al. ${ }^{12-15}$ o Zulueta y Bordons ${ }^{16}$, en los que se demuestra que la mayoría de los indicadores bibliométricos, especialmente la producción científica, han mejorado notablemente, lo que repercute favorablemente en la difusión de los avances en el sistema sanitario español. Este cambio de paradigma lleva a un proceso de constante evolución hacia la mejora de la calidad y el impacto, tal y como menciona Alfonso ${ }^{17}$.

No cabe duda de que la enfermería como ciencia debe desarrollarse fundamentando sus conocimientos en la evidencia científica. En esta línea, Benner ${ }^{18,19}$ señala la necesidad de sólidos conocimientos para que la trayectoria profesional de una enfermera se realice con éxito. Esta premisa también es compartida por Carrillo-Algarra et al. ${ }^{20}$ cuando afirman que es necesario un marco de desarrollo investigador para lograr una enfermería asistencial competente. Ahora bien, en todo este proceso es clave la existencia de una actitud favorable del profesional hacia la investigación. Esta actitud se puede evaluar a través de distintos instrumentos de medida, como los validados 
Tabla 1 Perfil de la muestra $(n=295)$

\begin{tabular}{|c|c|c|}
\hline Características & $\mathrm{n}$ & $\%$ \\
\hline \multicolumn{3}{|l|}{ Género } \\
\hline Hombre & 71 & 24,1 \\
\hline Mujer & 222 & 75,3 \\
\hline \multicolumn{3}{|l|}{ Edad } \\
\hline$<30$ años & 45 & 15,3 \\
\hline 30-40 años & 132 & 44,7 \\
\hline 41-50 años & 85 & 28,8 \\
\hline$>50$ años & 31 & 10,5 \\
\hline \multicolumn{3}{|l|}{ Puesto laboral } \\
\hline Enfermera & 273 & 92,5 \\
\hline Enfermero especialista & 18 & 6,1 \\
\hline EIR en formación & 3 & 1 \\
\hline \multicolumn{3}{|l|}{ Experiencia laboral } \\
\hline$<1$ año & 12 & 4,1 \\
\hline 1-5 años & 39 & 13,2 \\
\hline 6-10 años & 46 & 15,6 \\
\hline$>10$ años & 196 & 66,4 \\
\hline \multicolumn{3}{|l|}{ Servicio donde trabaja } \\
\hline Urgencias & 10 & 3,4 \\
\hline Quirófano & 29 & 9,8 \\
\hline $\mathrm{UCl}$ & 54 & 18,3 \\
\hline Hospitalización & 138 & 46,8 \\
\hline Consultas externas & 13 & 4,4 \\
\hline Hemodiálisis & 11 & 3,7 \\
\hline Paritorio & 8 & 2,7 \\
\hline Neonatos & 12 & 4,1 \\
\hline Hemodinámica & 3 & 1 \\
\hline Esterilización & 9 & 3,1 \\
\hline Reanimación & 1 & 0,3 \\
\hline URPA & 6 & 2 \\
\hline \multicolumn{3}{|l|}{ Experiencia investigadora } \\
\hline No & 225 & 76,3 \\
\hline Sí & 70 & 23,7 \\
\hline \multicolumn{3}{|l|}{ Méritos investigación } \\
\hline Doctorado & 1 & 1,1 \\
\hline Comunicaciones a congresos & 60 & 63,8 \\
\hline Artículos científicos & 22 & 23,4 \\
\hline Pertenece a algún grupo de investigación & 11 & 11,7 \\
\hline
\end{tabular}

por Corchon et al. ${ }^{21}$. Los estudios de Davies et al. ${ }^{22}$ y de Scott-Findlay y Golden-Biddle ${ }^{23}$ señalan que las enfermeras consideran que la investigación biomédica es muy útil para mejorar su capacidad asistencial y, por ello, el ambiente y la estructura laboral deben posibilitar su desarrollo principalmente en organizaciones complejas.

También el Consejo Internacional de Enfermería ${ }^{24}$ (CIE) considera necesario promover cambios en las dinámicas y estructuras de los grupos de investigación para que «contribuyan a generar nuevos conocimientos sobre lo que funciona y lo que no en relación con la implicación de las enfermeras en la configuración de las políticas de salud».

Por todo ello, es recomendable que se desarrollen políticas para promover la investigación entre el colectivo de la enfermería del hospital con perfil científico-investigador e incorporar investigadores básicos que ayuden a integrar la actividad asistencial y la investigadora. La idoneidad de estas políticas viene avalada por Asenjo et al. ${ }^{25}$ cuando establecen un paralelismo entre el índice de reputación sanitaria de los hospitales y su producción científica, que genera incluso la derivación y consultas de pacientes de un centro hacia otro.

El presente estudio pretende, en primer lugar, analizar la percepción de la enfermería sobre el actual sistema de investigación en el conjunto de su hospital. En segundo lugar, estudiar cómo varía dicha percepción en función del perfil investigador de la enfermera y de su nivel de satisfacción y compromiso con la organización en la que trabaja.

\section{Material y método}

Estudio descriptivo y transversal, realizado en el Complejo Hospitalario Universitario Materno Insular de Gran Canaria (CHUIMI) entre el colectivo de este centro. Se trabajó con una muestra de conveniencia de 295 enfermeras. Como criterios de inclusión se aceptó que los participantes tuviesen una experiencia en el medio hospitalario superior a 6 meses y desearan colaborar en el estudio de forma voluntaria. El procedimiento seguido para contactar con la población objeto de estudio ha sido solicitar personalmente a las supervisoras de Enfermería de cada uno de los servicios del CHUIMI que difundieran entre sus enfermeras el cuestionario. Dado que el procedimiento de recogida de cuestionarios se centralizó en las supervisoras de Enfermería, no fue necesario realizar recuerdos, aunque sí se les instaba en cada encuentro a motivar a su personal a que respondiese el cuestionario para conseguir una tasa elevada de respuesta.

La recogida de datos se realizó mediante un cuestionario anónimo, autoadministrado, adaptado al contexto y al colectivo, tomando como base el cuestionario desarrollado y validado por el Grupo de Expertos del III Foro de Ciencia de la Fundación Lilly, con el permiso del autor, Dr. Puerta ${ }^{5}$. La encuesta tenía por objeto recoger la percepción y valoración, tanto real como ideal, sobre el marco actual en el que se desarrolla la investigación en los hospitales. El cuestionario constaba de 19 ítems, cada uno de ellos medido mediante una escala tipo Likert donde el valor 1 representaba el mínimo y el valor 10, el máximo.

Para valorar el nivel de satisfacción y el nivel de compromiso se incluyeron en el cuestionario dos escalas tipo Likert como resultado de la revisión de la literatura, de 2 y 5 ítems, respectivamente, siendo la puntuación máxima en ambas escalas de 10 puntos. A partir de esta información, se calcularon los valores de las medias, resultando un valor de 7,3 para satisfacción y un valor de 5,6 para compromiso. Estas medias se tomaron como referencia para agrupar a los encuestados en dos grupos de satisfacción (baja y alta) y en dos de compromiso (bajo y alto).

En este trabajo se cataloga como personal investigador a quien estuviera en posesión del título de doctor, a quien fuese autor de comunicaciones presentadas a congresos y/o de artículos científicos $\mathrm{y}$, finalmente, a quien perteneciera a algún grupo de investigación. Para analizar la influencia del perfil investigador en la percepción de los encuestados se agruparon en dos grupos (no investigador y sí investigador). 
Los autores declaran no tener conflicto de intereses y manifiestan no haber utilizado datos de carácter personal en cumplimiento de la Ley Orgánica 44/1999 de Protección de Datos de Carácter Personal.

\section{Resultados}

La muestra de 295 enfermeras estuvo formada en su mayoría por mujeres (75,3\%), menores de 40 años (60\%) y con experiencia en el ámbito hospitalario superior a 10 años $(66,4 \%)$. El 46,8\% prestaba sus servicios en áreas de hospitalización y el $18,3 \%$ en la $\mathrm{UCl}$. El 76,3\% (225 casos) respondía a un perfil no investigador (véase tabla 1). Si se tiene en cuenta que la población objetivo ascendía a 2.070 enfermeras, este tamaño muestral implicó asumir un error de $\pm 5,39 \%$ para un intervalo de confianza del 95,5\%.

Los resultados de los análisis descriptivos llevados a cabo para conocer la percepción de la enfermería hacia la investigación indicaron que, en general, la percepción y valoración de la situación actual de la investigación en enfermería, el impacto de esta, su reconocimiento y su integración con la labor asistencial estaban muy por debajo de las puntuaciones ideales, que alcanzaron valores medios por encima de 8 en todos los ítems. Los resultados del estadístico $t$ de Student para valorar las diferencias entre la situación real y la ideal mostraron que las diferencias entre los valores medios reales y los ideales fueron, en todos los casos, estadísticamente significativas con valor $p<0,001$ y cercanas o muy superiores a 4 puntos para todos los ítems (véase tabla 2). Los valores registrados de este estadístico de diferencias de medias fueron aquellos que se derivaron de la existencia de igualdad o no de las varianzas de los grupos de acuerdo con la prueba de Levene, asumiendo un nivel de significación del $5 \%$.

De la información recogida en la tabla 3 se desprendió que la percepción del personal entrevistado difirió en función de si posee o no experiencia investigadora previa, siendo las enfermeras con perfil investigador $(n=70)$ las que valoraron más positivamente todos los ítems analizados, a excepción del último de ellos. Como se recoge en la tabla 1 , el personal con experiencia investigadora reconoció un total de 94 méritos investigadores.

Ahora bien, esta diferencia entre el personal de enfermería en función de su experiencia investigadora fue estadísticamente significativa solo en 5 de los 19 ítems, a un nivel de significación inferior o igual al 5\%. Cabe destacar que en ambos colectivos la percepción y valoración de la situación actual de la investigación en enfermería, el impacto de esta, su reconocimiento y su integración con la labor asistencial fueron muy bajos.

Cuando estudiamos las diferencias en el perfil investigador de las enfermeras de la $\mathrm{UCl}(18,3 \%, 54$ casos) respecto al resto, observamos que las primeras tenían una experiencia investigadora mayor $(31,8 \%$ vs. $21,5 \%)$. Sin embargo, no encontramos diferencias entre el personal de la UCI con el del resto de servicios en cuanto a la percepción y valoración de la investigación de la enfermería, a excepción de los dos últimos ítems, que preguntaban sobre el uso de sistemas de información en la investigación del hospital y la aplicabilidad de los resultados de la investigación. Fue por ello que puede afirmarse la existencia de una mayor vinculación de la enfermería de $\mathrm{UCl}$ con la labor investigadora.

Finalmente, en la tabla 4 se recogen los resultados de los análisis llevados a cabo para conocer la influencia de la satisfacción y el compromiso de la organización por parte del encuestado en su percepción real de la investigación de su entorno. En general, se puede afirmar que la valoración y la percepción del sistema de la investigación no difieren en función del nivel de satisfacción del personal de enfermería. En cambio, el personal más comprometido con la organización concedió mayor importancia a la participación de la enfermería en la investigación, solicitando un mayor reconocimiento de esta tarea por parte de compañeros y gerencia.

\section{Discusión}

De los datos extraídos del presente trabajo, podemos deducir que el personal de enfermería valora positivamente la investigación clínica, reconociendo a la vez que existe aún un largo camino por recorrer para poder aproximarse a una situación ideal. En nuestro caso, únicamente el $23,7 \%$ de la muestra reconoce tener experiencia investigadora, por tanto, podemos decir que existe un amplio margen de mejora al respecto de la formación en investigación en los centros sanitarios. En este sentido, Soriguer ${ }^{6}$ muestra «una preocupante autocomplacencia sobre la calidad de nuestro sistema sanitario», admite que «el fracaso de la investigación clínica de las instalaciones sanitarias es, probablemente, el mejor exponente de la (baja) calidad de la medicina española»; por tanto, la investigación se convierte en un instrumento a implementar para evaluar de forma real la calidad asistencial y mejorarla en lo que precise.

También hay que tener en cuenta que las enfermeras, en los últimos años, han avanzado a nivel académico de manera importante tras la publicación de los Reales Decretos (RD) $55 / 2005^{26}$ y $56 / 2005^{27}$, y el RD $1393 / 2007$ de 29 de octubre ${ }^{28}$, que posibilitó el acceso a los títulos de posgrado desde la misma rama de la enfermería, pudiendo, por tanto, acceder a una formación investigadora reglada desde los propios títulos oficiales. Una mayor y mejor calidad investigadora, como afirma Maciá Soler ${ }^{29}$, además de capacitar a las enfermeras en el área de la investigación, objetivo principal de los títulos de máster y doctorado, podría conducir a una mejor calidad asistencial. Este hecho también queda demostrado en nuestro estudio, ante las mejores puntuaciones respecto a la situación investigadora entre las enfermeras con experiencia investigadora.

Todo el personal de enfermería con o sin experiencia investigadora puntúa con valores muy bajos la investigación sanitaria actual, al igual que los hallazgos de otros autores $^{6,12,13,15}$, lo que nos debe hacer pensar en cómo mejorar más la investigación biomédica, que como dicen también otros autores $8,9,14,15$ ha mejorado en los años recientes, pero se encuentra todavía en un área susceptible de mejora. Así, por ejemplo, como comenta Amigó-Tadín ${ }^{30}$, se investiga en enfermería pero las publicaciones no tienen suficiente impacto al utilizar revistas no indexadas o de poca repercusión.

Por otra parte, se ha observado cómo el impacto de la investigación, su reconocimiento y su integración con 
Tabla 2 Diferencias en la percepción real e ideal hacia la investigación

\begin{tabular}{|c|c|c|c|c|c|c|c|}
\hline \multirow[t]{2}{*}{ Ítems } & \multicolumn{2}{|c|}{ Real } & \multicolumn{2}{|c|}{ Ideal } & \multirow[t]{2}{*}{$t$} & \multirow[t]{2}{*}{$p$} & \multirow[t]{2}{*}{ IC 95\% } \\
\hline & Media & DE & Media & $\mathrm{DE}$ & & & \\
\hline $\begin{array}{l}\text { La participación e } \\
\text { implicación del equipo } \\
\text { de enfermería y otro } \\
\text { personal sanitario } \\
\text { en investigación }\end{array}$ & 3,7 & 1,5 & 8,7 & 1,1 & 50,7 & 0,000 & $(-5,1 ;-4,7)$ \\
\hline $\begin{array}{l}\text { Algunas iniciativas } \\
\text { de investigación: } \\
\text { contratos FIS y becas } \\
\text { FUNCIS }\end{array}$ & 3,2 & 1,4 & 8,7 & 1,2 & 53,4 & 0,000 & $(-5,7 ;-5,3)$ \\
\hline $\begin{array}{l}\text { La participación } \\
\text { del personal de } \\
\text { enfermería en } \\
\text { investigación en su } \\
\text { hospital }\end{array}$ & 3,3 & 1,7 & 9,1 & 1,1 & 51,7 & 0,000 & $(-6,0,-5,5)$ \\
\hline $\begin{array}{l}\text { La necesidad de que, } \\
\text { en los hospitales } \\
\text { universitarios, } \\
\text { investiguen todos los } \\
\text { enfermeros y no solo } \\
\text { aquellos que hayan } \\
\text { demostrado interés } \\
\text { y capacidad para } \\
\text { hacerlo }\end{array}$ & 3,1 & 1,6 & 8,7 & 1,4 & 47,7 & 0,000 & $(-5,9 ;-5,5)$ \\
\hline $\begin{array}{l}\text { El impacto de la } \\
\text { investigación en el } \\
\text { hospital en la calidad } \\
\text { de la asistencia }\end{array}$ & 4,1 & 1,5 & 8,8 & 1,2 & 43,5 & 0,000 & $(-4,9 ;-4,5)$ \\
\hline $\begin{array}{l}\text { El impacto de la } \\
\text { investigación en el } \\
\text { hospital en la gestión } \\
\text { económica del centro }\end{array}$ & 3,3 & 1,5 & 8,9 & 1,3 & 47,7 & 0,000 & $(-5,8 ;-5,4)$ \\
\hline $\begin{array}{l}\text { El impacto social y global } \\
\text { de la investigación } \\
\text { biomédica en España }\end{array}$ & 3,5 & 1,7 & 9,1 & 1,2 & 44,7 & 0,000 & $(-5,8 ;-5,3)$ \\
\hline $\begin{array}{l}\text { La consideración de la } \\
\text { investigación como } \\
\text { una actividad } \\
\text { fundamental por parte } \\
\text { de los enfermeros }\end{array}$ & 3,7 & 1,5 & 8,8 & 1,2 & 47,2 & 0,000 & $(-5,3 ;-4,8)$ \\
\hline $\begin{array}{l}\text { Reconocimiento } \\
\text { explícito y apoyo de } \\
\text { los compañeros del } \\
\text { hospital a la labor } \\
\text { investigadora de los } \\
\text { enfermeros }\end{array}$ & 3,5 & 1,8 & 9,1 & 1,1 & 46,9 & 0,000 & $(-5,8 ;-5,3)$ \\
\hline $\begin{array}{l}\text { Reconocimiento } \\
\text { explícito en el hospital } \\
\text { de la labor } \\
\text { investigadora por } \\
\text { parte de la gerencia }\end{array}$ & 2,7 & 1,7 & 9,1 & 1,2 & 50,9 & 0,000 & $(-6,6 ;-6,1)$ \\
\hline $\begin{array}{l}\text { La coordinación de la } \\
\text { investigación del } \\
\text { hospital con otras } \\
\text { unidades u organismos } \\
\text { investigadores }\end{array}$ & 3,8 & 1,4 & 8,7 & 1,1 & 48,2 & 0,000 & $(-5,1 ;-4,7)$ \\
\hline
\end{tabular}


Tabla 2 (continuación)

\begin{tabular}{|c|c|c|c|c|c|c|c|}
\hline \multirow[t]{2}{*}{ Ítems } & \multicolumn{2}{|c|}{ Real } & \multicolumn{2}{|c|}{ Ideal } & \multirow[t]{2}{*}{$t$} & \multirow[t]{2}{*}{$p$} & \multirow[t]{2}{*}{ IC 95\% } \\
\hline & Media & DE & Media & DE & & & \\
\hline $\begin{array}{l}\text { La colaboración entre el } \\
\text { hospital y los centros } \\
\text { de salud en el terreno } \\
\text { de la investigación } \\
\text { biomédica }\end{array}$ & 3,1 & 1,5 & 8,9 & 1,1 & 53,2 & 0,000 & $(-6,0 ;-5,6)$ \\
\hline $\begin{array}{l}\text { Los ensayos clínicos } \\
\text { patrocinados por la } \\
\text { industria farmacéutica } \\
\text { en la investigación }\end{array}$ & 3,7 & 1,9 & 8,7 & 1,6 & 31,6 & 0,000 & $(-5,3 ;-4,7)$ \\
\hline $\begin{array}{l}\text { La utilidad de la relación } \\
\text { con la industria } \\
\text { farmacéutica o de } \\
\text { tecnología sanitaria } \\
\text { por los investigadores }\end{array}$ & 4,0 & 1,9 & 8,8 & 1,4 & 32,1 & 0,000 & $(-5,2 ;-4,6)$ \\
\hline $\begin{array}{l}\text { La integración y } \\
\text { articulación de la } \\
\text { asistencia, la } \\
\text { investigación y la } \\
\text { docencia en el hospital }\end{array}$ & 4,0 & 1,5 & 8,9 & 1,1 & 45,2 & 0,000 & $(-5,1 ;-4,7)$ \\
\hline $\begin{array}{l}\text { La consideración } \\
\text { explícita y } \\
\text { diferenciada de la } \\
\text { investigación en los } \\
\text { presupuestos de la } \\
\text { cartera de servicios } \\
\text { del hospital }\end{array}$ & 3,3 & 1,4 & 8,9 & 1,2 & 50,1 & 0,000 & $(-5,8 ;-5,4)$ \\
\hline $\begin{array}{l}\text { La existencia de una } \\
\text { estructura } \\
\text { organizativa en los } \\
\text { hospitales españoles } \\
\text { que potencie la labor } \\
\text { de investigación } \\
\text { clínica de los } \\
\text { enfermeros }\end{array}$ & 3,0 & 1,5 & 9,1 & 1,2 & 52,6 & 0,000 & $(-6,3 ;-5,9)$ \\
\hline $\begin{array}{l}\text { La utilización de } \\
\text { sistemas de } \\
\text { información, con la } \\
\text { debida protección } \\
\text { de datos, en la } \\
\text { investigación en el } \\
\text { hospital }\end{array}$ & 5,6 & 2,0 & 9,3 & 1,2 & 32,1 & 0,000 & $(-3,9 ;-3,4)$ \\
\hline $\begin{array}{l}\text { La traslacionali- } \\
\text { dad/aplicabilidad de } \\
\text { los resultados } \\
\text { de investigación } \\
\text { biomédica en el } \\
\text { hospital }\end{array}$ & 5,1 & 1,9 & 9,2 & 1,2 & 38,0 & 0,000 & $(-4,3 ;-3,9)$ \\
\hline
\end{tabular}

la labor asistencial, está muy por debajo de los valores ideales, lo que puede ser debido a varios factores, como los expresados por Moreno-Casbas et al. ${ }^{31}$ dentro de su «top three Barriers»; en primer lugar, no ser capaz de evaluar la calidad de la investigación; en segundo lugar, la insuficiente autoridad de las enfermeras para cambiar el cuidado y, por último, desconocer la evidencia. Otros autores, como
Ortuño-Soriano et al.32, afirman que las enfermeras están motivadas para investigar, pero que existen barreras formativas, laborales o familiares, que limitan el desarrollo de la competencia en investigación.

Nuestros resultados indican que la satisfacción no influye en la valoración de la investigación, sin embargo, el compromiso de las enfermeras con la institución sí es una 
Tabla 3 Influencia del perfil investigador en la percepción real hacia la investigación

\begin{tabular}{|c|c|c|c|c|c|c|c|}
\hline \multirow[t]{2}{*}{ Ítems } & \multicolumn{2}{|c|}{ No investigador $(n=225)$} & \multicolumn{2}{|c|}{ Sí investigador $(n=70)$} & \multirow[t]{2}{*}{$t$} & \multirow[t]{2}{*}{$p$} & \multirow[t]{2}{*}{ IC 95\% } \\
\hline & Media & DE & Media & DE & & & \\
\hline $\begin{array}{l}\text { La participación e } \\
\text { implicación del equipo } \\
\text { de enfermería y otro } \\
\text { personal sanitario en } \\
\text { investigación }\end{array}$ & 3,7 & 1,4 & 4,1 & 1,8 & 1,5 & 0,132 & $(-0,8 ; 0,1)$ \\
\hline $\begin{array}{l}\text { Algunas iniciativas } \\
\text { de investigación: } \\
\text { contratos FIS y becas } \\
\text { FUNCIS }\end{array}$ & 3,1 & 1,3 & 3,3 & 1,7 & 0,9 & 0,360 & $(-0,7 ; 0,2)$ \\
\hline $\begin{array}{l}\text { La participación } \\
\text { del personal de } \\
\text { enfermería en } \\
\text { investigación en su } \\
\text { hospital }\end{array}$ & 3,1 & 1,5 & 3,9 & 1,9 & 3,3 & 0,001 & $(-1,2 ;-0,3)$ \\
\hline $\begin{array}{l}\text { La necesidad de que, } \\
\text { en los hospitales } \\
\text { universitarios, } \\
\text { investiguen todos los } \\
\text { enfermeros y no solo } \\
\text { aquellos que hayan } \\
\text { demostrado interés } \\
\text { y capacidad para } \\
\text { hacerlo }\end{array}$ & 3,0 & 1,6 & 3,2 & 1,6 & 1,3 & 0,208 & $(-0,7 ; 0,2)$ \\
\hline $\begin{array}{l}\text { El impacto de la } \\
\text { investigación en el } \\
\text { hospital en la calidad } \\
\text { de la asistencia }\end{array}$ & 4,0 & 1,5 & 4,5 & 1,7 & 2,3 & 0,026 & $(-1,0 ;-0,1)$ \\
\hline $\begin{array}{l}\text { El impacto de la } \\
\text { investigación en el } \\
\text { hospital en la gestión } \\
\text { económica del centro }\end{array}$ & 3,3 & 1,4 & 3,6 & 1,9 & 1,3 & 0,191 & $(-0,8 ; 0,2)$ \\
\hline $\begin{array}{l}\text { El impacto social y global } \\
\text { de la investigación } \\
\text { biomédica en España }\end{array}$ & 3,4 & 1,6 & 3,9 & 2,2 & 2,0 & 0,051 & $(-1,1 ; 0,0)$ \\
\hline $\begin{array}{l}\text { La consideración de la } \\
\text { investigación como } \\
\text { una actividad } \\
\text { fundamental por parte } \\
\text { de los enfermeros }\end{array}$ & 3,6 & 1,4 & 4,0 & 1,9 & 1,4 & 0,162 & $(-0,8 ; 0,1)$ \\
\hline $\begin{array}{l}\text { Reconocimiento } \\
\text { explícito y apoyo de } \\
\text { los compañeros del } \\
\text { hospital a la labor } \\
\text { investigadora de los } \\
\text { enfermeros }\end{array}$ & 3,4 & 1,7 & 3,8 & 2,0 & 1,6 & 0,107 & $(-1,0 ; 0,1)$ \\
\hline $\begin{array}{l}\text { Reconocimiento } \\
\text { explícito en el hospital } \\
\text { de la labor } \\
\text { investigadora por } \\
\text { parte de la gerencia }\end{array}$ & 2,6 & 1,7 & 3,1 & 1,9 & 2,4 & 0,019 & $(-1,0 ;-0,1)$ \\
\hline $\begin{array}{l}\text { La coordinación de la } \\
\text { investigación del } \\
\text { hospital con otras } \\
\text { unidades u organismos } \\
\text { investigadores }\end{array}$ & 3,7 & 1,3 & 4,0 & 1,6 & 1,2 & 0,246 & $(-0,7 ; 0,2)$ \\
\hline
\end{tabular}


Tabla 3 (continuación)

\begin{tabular}{|c|c|c|c|c|c|c|c|}
\hline \multirow[t]{2}{*}{ Ítems } & \multicolumn{2}{|c|}{ No investigador $(n=225)$} & \multicolumn{2}{|c|}{ Sí investigador $(n=70)$} & \multirow[t]{2}{*}{$t$} & \multirow[t]{2}{*}{$p$} & \multirow[t]{2}{*}{ IC $95 \%$} \\
\hline & Media & $\mathrm{DE}$ & Media & $\mathrm{DE}$ & & & \\
\hline $\begin{array}{l}\text { La colaboración entre el } \\
\text { hospital y los centros } \\
\text { de salud en el terreno } \\
\text { de la investigación } \\
\text { biomédica }\end{array}$ & 3,1 & 1,4 & 3,2 & 1,7 & 0,6 & 0,570 & $(-0,5 ; 0,3)$ \\
\hline $\begin{array}{l}\text { Los ensayos clínicos } \\
\text { patrocinados por la } \\
\text { industria farmacéutica } \\
\text { en la investigación }\end{array}$ & 3,6 & 1,8 & 4,4 & 2,2 & 2,7 & 0,009 & $(-1,4 ;-0,2)$ \\
\hline $\begin{array}{l}\text { La utilidad de la relación } \\
\text { con la industria } \\
\text { farmacéutica o de } \\
\text { tecnología sanitaria } \\
\text { por los investigadores }\end{array}$ & 3,8 & 1,8 & 4,6 & 2,2 & 3,0 & 0,003 & $(-1,4 ;-0,3)$ \\
\hline $\begin{array}{l}\text { La integración y } \\
\text { articulación de la } \\
\text { asistencia, la } \\
\text { investigación y la } \\
\text { docencia en el hospital }\end{array}$ & 3,9 & 1,5 & 4,2 & 1,8 & 0,9 & 0,345 & $(-0,7 ; 0,2)$ \\
\hline $\begin{array}{l}\text { La consideración } \\
\text { explícita y } \\
\text { diferenciada de la } \\
\text { investigación en los } \\
\text { presupuestos de la } \\
\text { cartera de servicios } \\
\text { del hospital }\end{array}$ & 3,3 & 1,4 & 3,4 & 1,7 & 0,6 & 0,571 & $(-0,6 ; 0,3)$ \\
\hline $\begin{array}{l}\text { La existencia de una } \\
\text { estructura } \\
\text { organizativa en los } \\
\text { hospitales españoles } \\
\text { que potencie la labor } \\
\text { de investigación } \\
\text { clínica de los } \\
\text { enfermeros }\end{array}$ & 3,0 & 1,5 & 3,1 & 1,6 & 0,7 & 0,508 & $(-0,6 ; 0,3)$ \\
\hline $\begin{array}{l}\text { La utilización de } \\
\text { sistemas de } \\
\text { información, con la } \\
\text { debida protección de } \\
\text { datos, en la } \\
\text { investigación en el } \\
\text { hospital }\end{array}$ & 5,6 & 2,0 & 5,7 & 2,0 & 0,6 & 0,575 & $(-0,7 ; 0,4)$ \\
\hline $\begin{array}{l}\text { La traslacionali- } \\
\text { dad/aplicabilidad de } \\
\text { los resultados } \\
\text { de investigación } \\
\text { biomédica en el } \\
\text { hospital }\end{array}$ & 5,2 & 1,9 & 4,8 & 1,8 & 1,8 & 0,075 & $(0,0 ; 1,0)$ \\
\hline
\end{tabular}

faceta que les afecta de manera notable, posiblemente debido al compromiso social que representa la investigación. Por ello, se debe llevar a cabo una adecuada asignación de recursos para la investigación, ya que esta se debe visualizar como un coste-oportunidad, que viene determinado por el continuo avance sanitario y flujo de pacientes $^{33}$.
Ramos-Morcillo y Ruzafa-Martínez ${ }^{34}$ afirman que las enfermeras abordan los problemas asistenciales detectados desde un enfoque distinto que no aplica ninguna otra profesión sanitaria. Este hecho implica la necesidad de recibir apoyo de las instituciones de investigación nacional, que no siempre se acuerdan en sus proyectos y subvenciones de las líneas de investigación en cuidados de enfermería, lo cual 
Tabla 4 Influencia de la satisfacción y el compromiso en la percepción real hacia la investigación

\begin{tabular}{|c|c|c|c|c|c|c|c|c|c|c|c|c|c|c|}
\hline Ítems & \multicolumn{2}{|c|}{$\begin{array}{l}\text { Baja satisfacción } \\
\mathrm{n}=109(36,9 \%)\end{array}$} & \multicolumn{2}{|c|}{$\begin{array}{l}\text { Alta satisfacción } \\
\mathrm{n}=185(62,7 \%)\end{array}$} & $t$ & $p$ & IC 95\% & \multicolumn{2}{|c|}{$\begin{array}{l}\text { Bajo compromiso } \\
\mathrm{n}=164(55,6 \%)\end{array}$} & \multicolumn{2}{|c|}{$\begin{array}{l}\text { Alto compromiso } \\
n=128(43,4 \%)\end{array}$} & $t$ & $p$ & IC 95\% \\
\hline $\begin{array}{l}\text { La participación e } \\
\text { implicación del equipo } \\
\text { de enfermería en } \\
\text { investigación }\end{array}$ & 3,6 & 2,0 & 3,9 & 1,2 & 1,2 & 0,247 & $(-0,7 ; 0,2)$ & 3,8 & 1,3 & 3,7 & 1,9 & 0,5 & 0,652 & $(-0,3 ; 0,5)$ \\
\hline $\begin{array}{l}\text { Algunas iniciativas de } \\
\text { investigación: contratos } \\
\text { FIS y becas FUNCIS }\end{array}$ & 3,0 & 1,6 & 3,3 & 1,3 & 1,5 & 0,136 & $(-0,6 ; 0,1)$ & 3,3 & 1,2 & 3,1 & 1,7 & 0,8 & 0,415 & $(-0,2 ; 0,5)$ \\
\hline $\begin{array}{l}\text { La necesidad de que, en } \\
\text { los hospitales } \\
\text { universitarios, } \\
\text { investiguen todos los } \\
\text { enfermeros y no solo } \\
\text { aquellos que hayan } \\
\text { demostrado interés y } \\
\text { capacidad para hacerlo }\end{array}$ & 3,1 & 1,8 & 3,0 & 1,5 & 0,5 & 0,626 & $(-0,3 ; 0,5)$ & 2,9 & 1,4 & 3,3 & 1,8 & 2,3 & 0,023 & $\begin{array}{l}(-0,8 \\
-0,1)\end{array}$ \\
\hline $\begin{array}{l}\text { El impacto de la } \\
\text { investigación en el } \\
\text { hospital en la gestión } \\
\text { económica del centro }\end{array}$ & 3,4 & 1,9 & 3,4 & 1,3 & 0,3 & 0,789 & $(-0,4 ; 0,5)$ & 3,1 & 1,3 & 3,8 & 1,8 & 3,3 & 0,001 & $\begin{array}{l}(-1,0 \\
-0,3)\end{array}$ \\
\hline $\begin{array}{l}\text { El impacto social y global } \\
\text { de la investigación } \\
\text { biomédica en España }\end{array}$ & 3,6 & 2,0 & 3,5 & 1,6 & 0,5 & 0,600 & $(-0,3 ; 0,5)$ & 3,3 & 1,5 & 3,9 & 2,1 & 2,7 & 0,007 & $\begin{array}{l}(-1,0 \\
-0,2)\end{array}$ \\
\hline $\begin{array}{l}\text { Consideración de la } \\
\text { investigación como } \\
\text { actividad fundamental }\end{array}$ & 3,5 & 1,7 & 3,9 & 1,4 & 1,8 & 0,080 & $(-0,7 ; 0,0)$ & 3,7 & 1,1 & 3,8 & 1,9 & 0,8 & 0,443 & $(-0,5 ; 0,2)$ \\
\hline
\end{tabular}




\begin{tabular}{|c|c|c|c|c|c|c|c|c|c|c|c|c|c|c|}
\hline \multirow[t]{2}{*}{ Ítems } & \multicolumn{2}{|c|}{$\begin{array}{l}\text { Baja satisfacción } \\
\mathrm{n}=109(36,9 \%)\end{array}$} & \multicolumn{2}{|c|}{$\begin{array}{l}\text { Alta satisfacción } \\
\mathrm{n}=185(62,7 \%)\end{array}$} & \multirow[t]{2}{*}{$t$} & \multirow[t]{2}{*}{$p$} & \multirow[t]{2}{*}{ IC 95\% } & \multicolumn{2}{|c|}{$\begin{array}{l}\text { Bajo compromiso } \\
n=164(55,6 \%)\end{array}$} & \multicolumn{2}{|c|}{$\begin{array}{l}\text { Alto compromiso } \\
\mathrm{n}=128(43,4 \%)\end{array}$} & \multirow[t]{2}{*}{$t$} & \multirow[t]{2}{*}{$p$} & \multirow[t]{2}{*}{ IC 95\% } \\
\hline & Media & $\mathrm{DE}$ & Media & $\mathrm{DE}$ & & & & Media & $\mathrm{DE}$ & Media & $\mathrm{DE}$ & & & \\
\hline $\begin{array}{l}\text { Reconocimiento } \\
\text { explícito en el hospital } \\
\text { de la labor investigadora } \\
\text { por parte de la gerencia }\end{array}$ & 2,8 & 1,7 & 2,6 & 1,8 & 1,0 & 0,330 & $(-0,2 ; 0,6)$ & 2,4 & 1,5 & 3,1 & 2,0 & 2.9 & 0,004 & $\begin{array}{l}(-1,0 \\
-0,2)\end{array}$ \\
\hline $\begin{array}{l}\text { La coordinación de la } \\
\text { investigación del } \\
\text { hospital con otras } \\
\text { unidades u organismos }\end{array}$ & 3,5 & 1,5 & 3,9 & 1,3 & 2,5 & 0,015 & $\begin{array}{l}(-0,8 \\
-0,1)\end{array}$ & 3,8 & 1,1 & 3,8 & 1,7 & 0,1 & 0,941 & $(-0,3 ; 0,3)$ \\
\hline $\begin{array}{l}\text { La colaboración entre el } \\
\text { hospital y los centros de } \\
\text { salud en el terreno de la } \\
\text { investigación }\end{array}$ & 3,0 & 1,6 & 3,2 & 1,4 & 0,9 & 0,360 & $(-0,5 ; 0,2)$ & 3,1 & 1,2 & 3,2 & 1,8 & 1,0 & 0,328 & $(-0,5 ; 0,2)$ \\
\hline $\begin{array}{l}\text { La utilidad de la relación } \\
\text { con la industria } \\
\text { farmacéutica o de } \\
\text { tecnología sanitaria }\end{array}$ & 4,1 & 2,0 & 3,9 & 1,9 & 0,8 & 0,446 & $(-0,3 ; 0,7)$ & 3,9 & 1,7 & 4,1 & 2,2 & 0,9 & 0,369 & $(-0,7 ; 0,3)$ \\
\hline
\end{tabular}




\section{Tabla 4 (continuación)}

\begin{tabular}{|c|c|c|c|c|c|c|c|c|c|c|c|c|c|c|}
\hline \multirow[t]{2}{*}{ Ítems } & \multicolumn{2}{|c|}{$\begin{array}{l}\text { Baja satisfacción } \\
n=109(36,9 \%)\end{array}$} & \multicolumn{2}{|c|}{$\begin{array}{l}\text { Alta satisfacción } \\
\mathrm{n}=185(62,7 \%)\end{array}$} & \multirow[t]{2}{*}{$t$} & \multirow[t]{2}{*}{$p$} & \multirow[t]{2}{*}{ IC 95\% } & \multicolumn{2}{|c|}{$\begin{array}{l}\text { Bajo compromiso } \\
\mathrm{n}=164(55,6 \%)\end{array}$} & \multicolumn{2}{|c|}{$\begin{array}{l}\text { Alto compromiso } \\
\mathrm{n}=128(43,4 \%)\end{array}$} & \multirow[t]{2}{*}{$t$} & \multirow[t]{2}{*}{$p$} & \multirow[t]{2}{*}{ IC 95\% } \\
\hline & Media & DE & Media & $\mathrm{DE}$ & & & & Media & DE & Media & $\mathrm{DE}$ & & & \\
\hline $\begin{array}{l}\text { La integración y } \\
\text { articulación de la } \\
\text { asistencia, la } \\
\text { investigación y la } \\
\text { docencia en el hospital }\end{array}$ & 3,7 & 1,7 & 4,2 & 1,4 & 2,6 & 0,011 & $\begin{array}{l}(-0,9 \\
-0,1)\end{array}$ & 3,9 & 1,2 & 4,2 & 1,9 & 1,6 & 0,111 & $(-0,7 ; 0,1)$ \\
\hline $\begin{array}{l}\text { La consideración } \\
\text { explícita y diferenciada } \\
\text { de la investigación en los } \\
\text { presupuestos de la } \\
\text { cartera de servicios del } \\
\text { hospital }\end{array}$ & 3,3 & 1,7 & 3,4 & 1,3 & 0,6 & 0,568 & $(-0,5 ; 0,3)$ & 3,2 & 1,2 & 3,5 & 1,7 & 1,2 & 0,221 & $(-0,6 ; 0,1)$ \\
\hline $\begin{array}{l}\text { La existencia de una } \\
\text { estructura organizativa } \\
\text { en los hospitales } \\
\text { españoles que potencie } \\
\text { la labor de investigación } \\
\text { clínica de los enfermeros }\end{array}$ & 3,0 & 1,6 & 3,0 & 1,5 & 0,4 & 0,699 & $(-0,4 ; 0,3)$ & 2,9 & 1,4 & 3,2 & 1,6 & 1,5 & 0,135 & $(-0,6 ; 0,1)$ \\
\hline $\begin{array}{l}\text { La utilización de } \\
\text { sistemas de información, } \\
\text { con la debida protección } \\
\text { de datos, en la } \\
\text { investigación en el } \\
\text { hospital }\end{array}$ & 5,2 & 2,4 & 5,9 & 1,7 & 2,6 & 0,009 & $\begin{array}{l}(-1,2 \\
-0,2)\end{array}$ & 5,8 & 1,7 & 5,4 & 2,4 & 1,6 & 0,116 & $(-0,1 ; 0,9)$ \\
\hline $\begin{array}{l}\text { La traslacionali- } \\
\text { dad/aplicabilidad de los } \\
\text { resultados de } \\
\text { investigación biomédica }\end{array}$ & 4,3 & 2,1 & 5,6 & 1,7 & 5,2 & 0,000 & $\begin{array}{l}(-1,7 \\
-0,8)\end{array}$ & 5,7 & 1,6 & 4,5 & 2,0 & 5,3 & 0,000 & $(0,7 ; 1,6)$ \\
\hline
\end{tabular}


representa una dificultad añadida para que las enfermeras diseñen y realicen proyectos de investigación.

En cuanto a las limitaciones del estudio, en primer lugar, se ha limitado a una población concreta que es el personal de enfermería de un hospital, por consiguiente se debería ampliar la muestra, en futuros trabajos de investigación, a otros servicios asistenciales como son los extrahospitalarios. Y, en segundo lugar, el presente estudio se ha realizado solo en un hospital, por lo que en posteriores investigaciones debería ampliarse a otros hospitales.

Como conclusiones, creemos que, en primer lugar, las enfermeras asumen su rol investigador como parte de sus funciones como profesionales sanitarios.

En segundo lugar, bajo la perspectiva del propio personal de enfermería, la situación actual de la investigación es muy mejorable. Para ello, es fundamental que los profesionales dispongan de una red de apoyo desde las propias instituciones, las cuales deben favorecer la creación de una red de enfermeras y/o profesionales sanitarios de distintas categorías que cooperen en la planificación, ejecución, análisis, evaluación y publicación de trabajos de impacto.

Hemos objetivado una mayor vinculación de la enfermería de $\mathrm{UCl}$ con la labor investigadora, aunque no encontramos diferencias con el del resto de servicios en cuanto a la percepción y valoración de la investigación de la enfermería.

Por último, cabe decir que los resultados de este trabajo alumbran una oportunidad de mejora en un ámbito que, con el paso de los años, será cada vez más importante.

\section{Autoría}

Los autores han colaborado en la concepción y diseño del manuscrito, la recogida de datos, el análisis e interpretación de los datos y, por último, en la redacción, revisión y aprobación del manuscrito remitido.

\section{Conflicto de intereses}

No existen por parte de ninguno de los autores.

\section{Bibliografía}

1. Martín-Moreno J, Toharia J, Gutiérrez Fuentes JA. Evaluación y priorización de la investigación científica en España. El punto de vista de los investigadores. Med Clin (Barc). 2008;131 Supl. 5:12-9.

2. Ministerio de Sanidad y Consumo. Real Decreto 339/2004, de 27 de febrero, sobre acreditación de institutos de investigación sanitaria. BOE. 2004;11409-12.

3. Ministerio de Sanidad y Consumo. Orden SCO/1245/2006, de 18 de abril, por la que se desarrolla el Real Decreto 339/2004, de 27 de febrero, sobre acreditación de institutos de investigación sanitaria. BOE. 2006;16538-40.

4. Ministerio de la Presidencia. Real Decreto 279/2016, de 24 de junio, sobre acreditación de institutos de investigación biomédica o sanitaria. BOE. 2016;47272-83.

5. Puerta JL, Martín-Moreno JM, Bravo S, Gutiérrez-Fuentes JA. Valoración de la investigación que se realiza en los hospitales españoles. Rev Clin Esp. 2011;211:169-78.

6. Soriguer F. El fracaso de la investigación clínica en España. Med Clin (Barc). 2009;132:219-21.
7. Pons J, Sais C, Illa C, Méndez R, Suñen E, Casas M, et al. Is there an association between the quality of hospitals' research and their quality of care? J Health Serv Res Policy. 2010;15:204-9.

8. Miró O, Sesma J, Burillo-Putze G. La investigación en medicina de urgencias y emergencias. An Sist Sanit Navar. 2010;33 Supl. $1: 215-27$.

9. Vento Torres M, Villamar E, Botet F, González de Dios J, García-Muñoz F, Sáenz de Pipaón M. Encuesta sobre la actividad investigadora en los servicios de neonatología en España en el período 2000-2004. An Pediatr. 2007;67:301-8.

10. Font D, Gomis R, Trilla A, Bigorra J, Piqué JM, Rodés J. Organización y modelo de funcionamiento de las estructuras de investigación biomédica. Situación y retos de futuro. Med Clin (Barc). 2008;130:510-6.

11. Goldhamer MEJ, Cohen AP, Bates DW, Cook EF, Davis RB, Singer $\mathrm{DE}$, et al. Protecting an endangered species: training physicians to conduct clinical research. Acad Med. 2009;84:439-45.

12. Camí J, Fernández MT, Gómez-Caridad I. La producción científica española en biomedicina y salud. Un estudio a través del Science Citation Index (1986-1989). Med Clin (Barc). 1993;101:721-31.

13. Camí J, Suñén-Piñol E, Méndez-Vásquez R. Mapa bibliométrico de España 1994-2002: biomedicina y ciencias de la salud. Med Clin (Barc). 2005;124:93-101.

14. Camí J, Suñen E, Carbó JM, Coma L. Producción científica española en Biomedicina y Ciencias de la Salud. Mapa bibliométrico de la investigación realizada en España durante el período 1994-2000. Informe del Instituto de Salud Carlos III-Fondo de Investigación Sanitaria; 2002.

15. Camí J, Zulueta MA, Fernández MT, Bordons M, Gómez I. Producción científica española en biomedicina y ciencias de la salud durante el período 1990-1993 (Science Citation Index y Social Science Citation Index) y comparación con el período 1986-1989. Med Clin (Barc). 1997;109:481-96.

16. Zulueta MA, Bordons M. La producción científica española en el área cardiovascular a través del Science Citation Index (19901996). Rev Esp Cardiol. 1999;52:751-64.

17. Alfonso F. El duro peregrinaje de las revistas biomédicas españolas hacia la excelencia: ¿quién nos ayuda? Calidad, impacto y méritos de la investigación. Endocrinol Nutr. 2010;57:110-20.

18. Benner P. From novice to expert: excellence and power in clinical nursing practice. Prentice Hall; 2001. p. 307 p.

19. Benner P, Spichiger E, Wallhagen MI. Nursing as a caring practice from a phenomenological perspective. Scand J Caring Sci. 2005; 19:303-9.

20. Carrillo-Algarra AJ, García-Serrano L, Cárdenas-Orjuela CM, Díaz-Sánchez IR, Yabrudy-Wilches N. La filosofía de Patricia Benner y la práctica clínica. Enferm Glob. 2013;32:346-61.

21. Corchon S, Watson R, Arantzamendi M, Saracíbar M. Design and validation of an instrument to measure nursing research culture: The Nursing Research Questionnaire (NRQ). J Clin Nurs. 2010;19:217-26.

22. Davies J, Heyman B, Bryar R, Graffy J, Gunnell C, Lamb B, et al. The research potential of practice nurses. Health Soc Care Community. 2002;10:370-81.

23. Scott-Findlay S, Golden-Biddle K. Understanding how organizational culture shapes research use. J Nurs Adm. 2005;35:359-65.

24. Consejo Internacional de Enfermeras (CIE). Las enfermeras, una fuerza para el cambio: eficaces en cuidados, eficientes en costos. Ginebra, Suiza: CIE; 2015, 1-70 p.

25. Asenjo MA, Bertrán MJ, Guinovart C, Llach M, Prat A, Trilla A. Análisis de la reputación de los hospitales españoles: relación con su producción científica en cuatro especialidades. Med Clin (Barc). 2006;126:768-70.

26. Ministerio de Educación y Ciencia. Real Decreto 55/2005 de 21 de enero, por el que establece la estructura de las enseñanzas 
universitarias y se regulan los estudios universitarios oficiales de Grado. BOE. 2010;2842-6.

27. Ministerio de Educación y Ciencia. Real Decreto 56/2005, de 21 de enero, por el que se regulan los estudios universitarios oficiales de Posgrado. BOE. 2005;21:2846-51.

28. Ministerio de Educación y Ciencia. Real Decreto 1393/2007, de 29 de octubre, por el que se establece la ordenación de las enseñanzas universitarias oficiales. BOE. 2007;44037-48.

29. Maciá Soler L. Investigación en enfermería. Enferm Clin. 2008;18:287-8.

30. Amigó-Tadín M. La investigación de la enfermería de urgencias en España a través de la base de datos CUIDEN (200-2005). Emergencias. 2008;20:299-307.

31. Moreno-Casbas T, Fuentelsaz-Gallego C, de Miguel ÁG, González-María E, Clarke SP. Spanish nurses' attitudes towards research and perceived barriers and facilitators of research utilisation: a comparative survey of nurses with and without experience as principal investigators. J Clin Nurs. 2011;20:1936-47.

32. Ortuño-Soriano I, Posada-Moreno P, Fernández-del-Palacio E. Actitud y motivación frente a la investigación en un nuevo marco de oportunidad para los profesionales de enfermería. Index Enferm. 2013;22:132-6.

33. Artells Herrero JJ. Estrategia y asignación de recursos en la investigación biomédica. Gac Sanit. 2000;14:391-7.

34. Ramos-Morcillo AJ, Ruzafa-Martínez M. Investigación enfermera y políticas públicas de salud. De la «enfermeraginia» a la «enfermerología». Enferm Clin. 2017;27:141-3. 